Geopolítica(s) Revista de estudios sobre espacio y poder ISSN: 2172-3958

\title{
Comentario. Releer a Bowman, repensar la Geopolítica ${ }^{1}$
}

\author{
Juan Agulló2
}

Recibido: 15 de agosto de 2020 / Aceptado: 24 de septiembre de 2020

Resumen. Este artículo comenta Geography versus Geopolitics, redactado en 1942 por Isaiah Bowman y publicado por la Geographical Review. En estas líneas se argumenta que dicho texto, pese a ser producto de su militancia en la Geografía y por encima de ello, de un contexto político complejo, si es releído con una perspectiva crítica, puede ser considerado un interesante referente para la Geopolítica contemporánea. De hecho, lo que Bowman pretendió con su trabajo fue deconstruir la vieja Geopolitik alemana, una tradición ideológica que condensó, en sus supuestos y procedimientos, la mayor parte de deficiencias metodológicas del pensamiento clásico, todavía detectables en algunas formas actuales de concebir la Geopolítica.

Palabras clave: Alemania; Bowman; Geografía; Geopolítica; Segunda Guerra Mundial.

\section{[en] Commentary. Re-reading Bowman, Re-thinking Geopolitics}

\begin{abstract}
This paper comments Geography versus Geopolitics, written in 1942 by Isaiah Bowman and published by the Geographical Review. In these lines it is argued that the aforementioned text, despite being a product of his geographic activism and above it, the result of a complex political context, if reread in a critical view could be considered as an interesting reference for contemporary Geopolitics. In fact, what Bowman intended with his work was to deconstruct the old German Geopolitik, an ideological tradition that condensed, in its assumptions and in its procedures, most of the methodological deficiencies of Classical Thought, still detectable in some current ways of conceiving Geopolitics.
\end{abstract}

Keywords: Germany; Bowman; Geography; Geopolitics; World War II.

\section{[pt] Comentário. Relendo o Bowman, repensando a Geopolítica}

Resumo. Esse paper comenta Geography versus Geopolitics, escrito em 1942 por Isaiah Bowman e publicado pela Geographical Review. A colocação dessas linhas é que aquele texto, apesar de ser produto do seu ativismo geográfico e para além disso, de um contexto político complexo, se relido em um olhar crítico pode ser considerado uma referência interessante para a Geopolítica contemporânea. Alias, o intuito do Bowman com o seu trabalho foi desconstruir a velha Geopolitik alemã, uma tradição ideológica que sintetizou, nos seus pressupostos e procedimentos, a maioria dos deficiências me-

1 [Nota de la redacción] Comentario sobre el texto de Isaiah Bowman: "Geography vs. Geopolitics", publicado originalmente en Geographical Review, en octubre de 1942, vol. 32, núm. 4, pp. 646-658.

2 Instituto Latino-Americano de Economía, Sociedade e Política de la UNILA, Foz de Iguaçu (Brasil).

E-mail: juan.agullo@unila.edu.br 
todológicas do Pensamento Clássico que ainda influenciam algumas formas atuais de entender a Geopolítica.

Palavras-chave: Alemanha; Bowman; Geografia; Geopolítica; Segunda Guerra Mundial.

Sumario. Introducción. 1. Deconstruyendo el (con)texto. 2. (Re)contextualizando las críticas. Referencias.

Cómo citar: Agulló, J. (2020). "Comentario. Releer a Bowman, repensar la Geopolítica”. Geopolítica(s). Revista de estudios sobre espacio y poder, 11(2), 379-385.

\section{Introducción}

Releer Geography vs. Geopolitics tiene mucho sentido casi ochenta años después de su publicación. Isaiah Bowman (1878-1950) su autor, fue protagonista de algunos eventos que parece oportuno evocar en tiempos, como los actuales, de incertidumbre: como académico de prestigio y ejerciendo de consultor fue un espectador privilegiado de las dos guerras mundiales además de "arquitecto principal" de la ONU (Smith, 2003). Asistió, también, a una agonía epistemológica de la Geopolítica que, a mediados del siglo XX, pareció casi definitiva. Sin embargo y a pesar de que mediante textos como el aquí traducido Bowman pretendió contribuir a su descabello, en realidad acabó proporcionándole, de forma indirecta, munición argumental a su resurrección crítica, ocurrida mucho tiempo después de su fallecimiento.

Nos encontramos, no en vano, ante uno de los trabajos más (re)conocidos de un autor tan contradictorio y controvertido como trascendente. Bowman, pese a no gozar de mucho predicamento fuera del ámbito anglosajón contemporáneo, constituye un referente insoslayable de la historia del pensamiento "geopolítico". El artículo que aquí se presenta contó en el momento de su publicación, en plena Segunda Guerra Mundial, con unos niveles de distribución y de discusión poco comunes para un texto académico. Neil Smith describió el inusual contexto que rodeó al escrito en cuestión:

Bowman se aseguró de que su paper tuviera amplia distribución. Como de costumbre, distribuyó copias, no solo a geógrafos y a otros académicos, incluidos Weigert y Spykman, sino a líderes empresariales y políticos. Envió copias a los principales miembros del Departamento de Estado con los que ahora trabajaba como asesor e incluso, al vicepresidente Henry Wallace (Smith, 1984, p.73).

Por eso parece lógico preguntarse por la obliteración posterior del texto en cuestión. ¿Por qué ocurrió? Es muy probable que tuviera que ver con el sutil pero implacable manto académico de silencio que se cernió sobre la Geopolítica (y hasta cierto punto, incluso, sobre la propia Geografía) durante la Guerra Fría ${ }^{3}$. La velada acusación que pesó sobre ambas, especialmente en Estados Unidos, fue haber proporcionado soporte intelectual a las ansias expansionistas de la Alemania Nazi (y de paso, a las del Japón Imperial, citado explícitamente en el texto y a las de la Ita-

3 La prestigiosa Universidad de Harvard llegó a cerrar su Facultad de Geografía entre 1947 y 1951. 
lia Fascista, no mencionada aquí por Bowman) y de haberlo hecho, además, a partir de una supuesta falta de honestidad académica y de rigor metodológico que habría habilitado a sus detractores para definirla, durante decenios, como una "pseudociencia".

Contempladas desde una actualidad asaeteada por el relativismo científico, las Fake News (Brown, 2019) y una práctica Geopolítica todavía excesivamente polisémica, querellas intelectuales de este porte, interpelan. A mediados del siglo XX, en la época en la que Bowman redactó este texto, era relativamente frecuente que conspicuos representantes del pensamiento geográfico anglosajón (como el propio Mackinder) e incluso francófono (como Jacques Ancel) criticaran duramente las bases epistemológicas de la Geopolitik alemana.

Bowman, en ese contexto intelectual y con Estados Unidos recién incorporado a la contienda, procedió sin atajos. En su texto, deconstruyó, razonó y disparó con argumentos contundentes contra lo que consideraba una tradición anclada en valores morales que presuponían una manera diferente de entender la realidad: acusó abiertamente al pensamiento alemán de "misticismo"; denunció su etnocentrismo sistemático; evidenció sus inexcusables carencias metodológicas; le afeó los términos, entre groseros e interesados, en los que según él había venido haciendo uso de la cartografía; subrayó sus relaciones, entre incestuosas e idealistas, con las políticas públicas y se atrevió, sobre todas las cosas, con el hasta entonces sacrosanto y prácticamente indiscutido principio del Lebensraum recurrente coartada "pseudocientífica", en términos del propio Bowman, del expansionismo alemán.

\section{Deconstruyendo el (con)texto}

El texto en cuestión tiene cerca de seis mil palabras. Importa su fondo aunque, también, sus formas. Hay que considerar, además, su excepcional marco de desarrollo: Bowman lo redactó en 1942, un año después de la entrada de Estados Unidos en la Segunda Guerra Mundial. Un momento muy delicado en el que, según la historiografía, todas las opciones bélicas eran todavía posibles. Este texto en ese contexto, lo recuerda nuevamente Smith (1984) tenía intenciones y ambiciones: se trataba, fundamentalmente, de restarle legitimidad al enemigo nazi al tiempo que se dotaba de racionalidad a la decisión de Washington de irrumpir en la contienda.

En Estados Unidos, es importante subrayarlo pues también en este ámbito se cernió durante mucho tiempo otro incómodo manto de silencio, había existido un consistente lobby pro-nazi desarticulado meses antes de que Bowman redactara su artículo (Bell, 1970). Uno de los legados más inquietantes de ese pequeño pero influyente grupo había sido una campaña a favor de la no alineación que, debido a que había llegado a conectar con el hasta entonces tradicional aislacionismo estadounidense, había sido bien vista por Berlín. Existía, por tanto, un ejercicio pendiente de seducción política y patriótica sutilmente asociado a la situación. Bowman, teorizándolo, trataba de tomar posiciones y por supuesto, de publicitarlas desde su área de confort: así como, el mismo año en el que escribió el texto, había presionado para que se creara en la Universidad de la que era Rector (la John Hopkins, de Baltimore) un laboratorio de Física Aplicada — que, al parecer, terminó 
influyendo positivamente en el desarrollo técnico del esfuerzo bélico — a nivel más político, publicó el texto que aquí se presenta.

El paper en cuestión es por tanto abiertamente militante aunque, al mismo tiempo, intenta no ser un panfleto. No debe olvidarse que fue publicado por la prestigiosa Geographical Review y que el propio Bowman consideraba a la Geografía, a diferencia de la Geopolítica - y ese es uno de los argumentos en los que más insiste - una disciplina 'científica' (cuando habla de la Geopolitik cita, con ironía cáustica, una frase del viejo Rudolf Kjéllen definiendo la política alemana: "un misticismo en el que la ciencia cesa y la creencia comienza").

En paralelo, para comprender este ensayo en todas sus dimensiones, es importante considerar que, como subraya Ó Tuathail (1996) en el momento de su redacción concurrieron otros elementos, no estrictamente académicos ni "oficiales", que también deben ser tenidos en cuenta. Por ejemplo, que el 'activismo' de Bowman había venido canalizándose desde el final de la Primera Guerra Mundial, a través de instituciones como el todopoderoso Council on Foreign Relations, que él mismo contribuyó a fundar en $1921^{4}$. Lo suyo más que 'activismo' era, por consiguiente, un cabildeo de alto nivel que tendía a materializarse con "abogados, banqueros y académicos de la Costa Este" (ibid., 1996, p.120). No debe sorprender por ello que Steven Seegel (2018) se refiera con sarcasmo a los "personal bonds" ("vínculos personales") de Bowman con personajes como los citados o que Neil Smith (2003) hable de él, simplemente, como "el geógrafo de Roosevelt". Menos aún, las constantes referencias de Bowman al comercio como un posible eje "cooperativo" de las relaciones internacionales algo que, tiempo después, terminaría convirtiéndose en una política de Estado para Washington.

Sea como fuere, volviendo al texto en cuestión y considerando el distinguido público al que estaba dirigido resulta lógico que, los argumentos desplegados en el mismo sean rigurosos pero que, sobre todo, vengan adornados por un lenguaje $a d$ hoc: se trataba ni más ni menos que de deconstruir en términos epistemológicamente convincentes y ante actores intelectualmente preparados y con capacidad real de decisión en los Estados Unidos de los años 1940, la que Bowman identificaba como una de las mayores debilidades ideológicas de la Alemania Nazi. Algunas citas literales hablan, en ese sentido, por sí solas: 1) "poner los hechos en serie y sacarse de la manga reglas mnemotécnicas no puede ser considerado ni una contribución al conocimiento ni a la ciencia"; 2) "no podemos, simplemente, voltear el mapa y reorganizar sus partes como si fuéramos libres para plantar pueblos en territorios baldíos", y 3) "cuando Haushofer, acepta los planteamientos mackinderianos pero entiende la lucha por el Heartland eurasiático como una fase en la lucha por el control del mundo frisa el absurdo".

Como se puede comprobar, la claridad crítica con la que procede el geógrafo canadiense naturalizado estadounidense, constituye uno de los motivos principales por los que Geography vs. Geopolitics sigue siendo rescatable ocho décadas después. El texto en cuestión pergeña, en efecto, una cuidadosa anatomía de la Geopolitik alemana después de realizar, eso sí, una introducción de cara a la galería (dedi-

$4 \quad$ El Council on Foreign Relations es un lobby estadounidense, que tiene un estatuto similar al de una fundación y funciona como Think Tank centrado en asuntos internacionales y política exterior de Estados Unidos. Desde sus inicios ha tratado de ser, no solo una instancia de análisis y producción de conocimiento sino de encuentro, intercambio y debate entre los miembros de la élite política y económica del país. 
cada a "los principios de la democracia" y a las políticas del "mal vecino", es decir, de Alemania en Europa y del "buen vecino", o sea, de Estados Unidos en América) que, aunque es probable que, considerando las intenciones extra-académicas del texto, fuera redactada para conectar con el Zeitgeist (incluyendo ahí los paternalistas guiños sobre América Latina) también convirtió al ensayo en cuestión en objeto de algunas críticas posteriores que tendieron a centrarse, precisamente, en lo que se supone que su autor condenaba: toda forma de militancia en valores dogmáticos, asociada a un texto "científico" (Smith, 1984).

Al margen de consideraciones que, de todos modos, siguen proporcionando materia prima para la reflexión, Bowman teje una crítica razonada de la que, todavía hoy en día, siguen siendo dignos de reseñar sus elementos medulares. Básicamente plantea que, en términos generales, la Geopolitik - a diferencia de la que él llama 'Geografía científica' - tiende a partir con demasiada frecuencia de interpretaciones "distorsionadas" de la historia y/o de la realidad internacional acríticamente asumidas y sugiere además que dicha desviación se concreta con recurrencia en: 1) la persistencia de prejuicios (generalmente basados en elementos ideológicos como el patriotismo o el supremacismo, su inquietante reverso); 2) planteamientos descontextualizados y 3) causalidades poco rigurosas. Su curioso ejemplo/anécdota del Millonésimo Atlas de Hispanoamérica está encaminado, en la práctica, a transmitir la idea de que las cosas también pueden hacerse (y no solo pensarse) de forma diferente.

El texto está atravesado, además, por algunas otras consideraciones sobre la Geopolitik que vale la pena repasar debido a su trascendencia. Bowman realiza, por ejemplo, una interesante deconstrucción de los llamados "Inexorables Geopolíticos" como símbolos de un determinismo que, desde su perspectiva, no solo resulta políticamente peligroso sino científicamente inconsistente. Habla, también, de mapamundis que abarcan hasta los confines de la tierra - utilizando, curiosamente, el muy geopolítico término de Rimland, popularizado un par de años después por Spykman (1944) - y al hacerlo desliza las limitaciones epistemológicas de una Geopolítica circunscrita a los escenarios clásicos (cita expresamente, más o menos en contraposición a "Europa y Japón", lugares todavía hoy tan remotos y exóticos como Port Moresby, la Isla de Mayotte o Dutch Harbor). Realiza, por último y para terminar, una interesante referencia al sentido mismo del análisis geopolítico que es descrito, más que como una herramienta necesariamente finalista de política pública, como un medio y una estrategia de comprensión rigurosa del mundo.

\section{2. (Re)contextualizando las críticas}

Geography vs. Geopolitics es, a todos los niveles, un producto de su tiempo. Sin embargo, no debe ser considerado un fósil. De hecho, en el contexto actual, está muy vivo; es muy rescatable; toca muchas fibras todavía sensibles. Constituye, en definitiva, no solo un atractivo testimonio directo de un contexto histórico inflexivo sino también, a nivel más práctico, un ejemplo concreto de cómo pueden ser deconstruidos planteamientos reaccionarios que proporcionan interpretaciones sesgadas de la realidad internacional. De eso, lamentablemente, se sabe bastante en un momento como el actual. La receta, según Bowman, es simple: para desenmascarar 
la demagogia y la falta de honestidad interpretativa, es necesario proceder con compromiso científico y espíritu forense: desarticulando la estructura última de razonamientos sin sustento; identificando prejuicios ocultos y rebatiendo determinismos sin sentido.

El espíritu de su texto, de hecho, no debiera ser entendido en un contexto como el actual a partir de su literalidad más estricta. Ateniéndose a la ruptura epistemológica que, en la década de los 1990, protagonizaron autores como el ya citado Ó Tutathail, más que como un Geography vs. Geopolitics literal, quizás este ensayo podría/debería ser visto como un 'Geopolítica Crítica versus Geopolítica Clásica'. En otros términos: como un campo de problematización que aunque pudiera estar trufado de errores siempre está más abierto y es más flexible y consciente de la importancia del rigor, que el sistema de pensamiento cerrado, dogmático, ideologizado y plagado de inconsistencias científicas al que se contrapone.

Hay en ese sentido una afirmación de Ó Tuathail que describe a la perfección los ejes de lo que para él constituía, en 1996, la esencia del planteamiento de la Critical Geopolitics:

La Geopolítica Crítica no debe ser entendida como una especie de Teoría General de la Geopolítica o como una negación de la misma. Se trata, únicamente, de una aproximación que busca contextualizar, deconstruyéndolos, aquellos fenómenos/conocimientos que se analizan. La Geopolítica Crítica es, en cierto modo, una estrategia parasitaria que solo puede ser entendida como una forma de revisión/actualización crítica de la Geopolítica. Es posible establecer un paralelismo con técnicas militares. De hecho, a diferencia de la vieja Geopolítica Imperial que, en realidad, no era más que una estrategia de conocimiento con fines prácticos, la Geopolítica Crítica es una táctica de conocimiento con fines analíticos. Esta última solo funciona dentro de los parámetros conceptuales de la Geopolítica tradicional al punto de que de ella es de quien toma las herramientas necesarias para la deconstrucción (1996, p.53).

Dicho de otra manera y recuperando el hilo argumental: el texto de Bowman puede ser considerado como un despliegue de agudas observaciones e incluso, de herramientas metodológicas que anticiparon, a diversos niveles, al sistematizado instrumental de ruptura proporcionado décadas después por autores como el ya citado Ó Tuathail (pero, también, por otros referentes de la geopolítica contemporánea como John Agnew o Simon Dalby) con el objeto de "trabajar incorporando los parámetros que definirían aquello que, supuestamente, ya debería haberse identificado y definido como geopolítica" (ibid., 1996, p.48).

Casi todas las observaciones de fondo que Bowman realiza en este texto tienen, de hecho, algo que ver con eso: trabajo cooperativo, eliminación de prejuicios (comenzando por el determinismo); ampliación del campo de problematización; contextualización del objeto de estudio; establecimiento riguroso de causalidades y negación del elemento positivista (e incluso, estrictamente disciplinar) de la Geopolítica. Existe además, en paralelo, una línea de continuidad más o menos clara con las sugerencias concretas que décadas después realizó la Critical Geopolitics con el objeto de enriquecer, epistemológicamente hablando, el aparato crítico de la Geopolítica: superación del etnocentrismo y del Estado-centrismo; discusión de las 
representaciones; desterritorialización del objeto de estudio; etc. Todas ellas características de un método que, por cierto, brilló por su ausencia —al menos académicamente hablando - durante la Guerra Fría, en un contexto de bipolaridad.

Todavía queda, para terminar, una última observación que realizar: es posible (re)leer a Bowman, casi ochenta años después, proporcionándole sentido desde nuestro propio entorno y no solo por el envenenado guiño que Bowman realiza a nuestra región en su texto sino porque al final es cierto que en la América Latina contemporánea, con la Geopolítica Crítica, sucede algo que suena parecido a lo que los anglosajones denunciaban en relación a su propio contexto a finales del siglo $\mathrm{XX}$ :

Se le suele mencionar (e incluso, supuestamente, practicar) con fruición aunque, casi nunca, se problematiza como campo de conocimiento. De hecho aún no ha habido una discusión teórica sólida en relación a su naturaleza y mucho menos, en relación a lo que sería una Geopolítica Crítica (ibid., 1996, p.48).

Pensado a partir de una perspectiva como la que acaba de ser expuesta, el ensayo de Bowman podría fungir como uno de los textos de referencia de un debate que complementaría tanto al reciente boom académico de la Geopolítica, como a los notables avances críticos que, amparados en una rica y heterogénea tradición preexistente de pensamiento, se han venido dando en dicho ámbito en América Latina a lo largo de las últimas décadas. El mundo anglosajón hace más de veinte años que problematizando la Geopolítica, la (re)piensa. Una práctica sugerente para la que hay materia prima.

\section{Referencias}

Bell, L. V. (1970). The failure of Nazism in America. The German American Bund, 19361941. Political Science Quarterly, 85(4), 585-599. Recuperado de www.jstor.org/stable/2147597

Brown, É. (2019). Fake News' and conceptual Ethics. Journal of Ethics and Social Philosophy, 16(2), 144-154 DOI: 10.26556/jesp.v16i2.648

Seegel, S. (2018). Map Men: Transnational Lives and Deaths of Geographers in the Making of East Central Europe. Chicaho, Il: University of Chicago Press.

Smith, N. (1984). Political geographers of the past. Isaiah Bowman: Political Geography and Geopolitics. Political Geography Quarterly, 3(1), 69-76

Smith, N. (2003). American Empire. Roosevelt's Geographer and the prelude to Globalization. Berkeley, CA: University of California Press.

Spykman, N. (1944). The Geography of the Peace. New York, NY: Harcourt, Brace and Company

Ó Tuathail, G. (1996). Critical Geopolitics. The Politics of writing Global Space. London: Routledge. 